\title{
The age of the Sun and the relativistic corrections in the EOS
}

\author{
A. Bonanno ${ }^{1}$, H. Schlattl ${ }^{2,3}$, and L. Paternò ${ }^{3}$ \\ 1 INAF - Osservatorio Astrofisico di Catania, Città Universitaria, 95123 Catania, Italy \\ 2 Astrophysics Research Institute, Liverpool John Moores University, Twelve Quays House, Egerton Wharf, \\ Birkenhead CH41 1LD, UK \\ ${ }^{3}$ Dipartimento di Fisica e Astronomia dell'Università, Sezione Astrofisica, Città Universitaria, 95123 Catania, Italy
}

Received 18 April 2002 / Accepted 13 May 2002

\begin{abstract}
We show that the inclusion of special relativistic corrections in the revised OPAL and MHD equations of state has a significant impact on the helioseismic determination of the solar age. Models with relativistic corrections included lead to a reduction of about 0.05-0.08 Gyr with respect to those obtained with the old OPAL or MHD EOS. Our best-fit value is $t_{\text {seis }}=(4.57 \pm 0.11)$ Gyr which is in remarkably good agreement with the meteoritic value for the solar age. We argue that the inclusion of relativistic corrections is important for probing the evolutionary state of a star by means of the small frequency separations $\delta v_{\ell, n}=v_{\ell, n}-v_{\ell+2, n-1}$, for spherical harmonic degrees $\ell=0,1$ and radial order $n \gg \ell$.
\end{abstract}

Key words. Sun: interior - Sun: oscillations - equation of state

\section{Introduction}

The possibility of using helioseismology to constrain the solar age has been discussed by several authors in the past. Very recently Dziembowski et al. (1999) have shown that the most robust and accurate method is provided by the small frequency separation analysis (SFSA), $\delta v_{\ell, n}=v_{\ell, n}-v_{\ell+2, n-1}$, for spherical harmonic degrees $\ell=0,1$ and radial order $n \gg \ell$ (Tassoul 1980).

The important property of this quantity is its strong sensitivity to the sound-speed gradient near the solar centre and its weak dependence on the details of the treatment of the outer layers. Despite our ignorance of a reliable convection model for the solar envelope we are therefore able to verify how well our models are able to reproduce the deep radiative regions, in particular the solar core. Since the properties of the core are mainly determined by the present central hydrogen abundance, and the latter is influenced by the solar age, SFSA is a reliable tool to examine the seismic age of the Sun.

Adopting the OPAL equation of state (Rogers et al. 1996) a seismic age of $(4.66 \pm 0.11)$ Gyr has been obtained by Dziembowski et al. (1999), which is consistent with the meteoritic age (4.57 \pm 0.02$)$ Gyr of Bahcall et al. (1995).

The aim of this paper is to show that an important ingredient in this type of analysis is the usage of an accurate equation of state (EOS). In particular, by the inclusion of the special relativistic corrections, like in the updated version of the OPAL EOS, the helioseismic age of the Sun is reduced to

Send offprint requests to: A. Bonanno,

e-mail: abo@ct.astro.it
$(4.57 \pm 0.11)$ Gyr, which is in remarkable agreement with the meteoritic value.

Elliott \& Kosovichev (1998) have demonstrated that the inclusion of relativistic corrections in the EOS leads to a better agreement between the solar models and the seismic Sun. By inverting SOI-MDI/SOHO $p$-mode frequencies they found that the solar adiabatic exponent $\Gamma_{1}$ is much better reproduced by solar models including the relativistic contribution to the Fermi-Dirac statistics. Since the improved EOS causes a decrease of $0.2 \%$ in the adiabatic index $\Gamma_{1}$ in the solar centre, the sound speed $\left(\propto \sqrt{\Gamma_{1}}\right)$ is reduced by about $0.1 \%$. Therefore, the influence of the relativistic corrections should also be visible in the small frequency separations $\delta v_{\ell, n}$. Indeed, Bonanno et al. (2001) have found that including this effect in the value of $\Gamma_{1}$ improves the agreement in $\delta v_{\ell, n}$ between solar models and observations, thereby confirming the results of Elliott \& Kosovichev (1998).

In addition to the age, the central hydrogen abundance is also crucially dependent on the precise value of $S_{\mathrm{pp}}(0)$, the zero-energy astrophysical $S$-factor for the proton-proton fusion cross section. Schlattl et al. (1999) and Antia \& Chitre (1999) have shown, using the old version of the OPAL EOS, that an increase of $S_{\mathrm{pp}}(0)$ by about $4 \%$ with respect to Adelberger et al.'s (1998) value yields a better agreement with the observed frequencies for an age of $4.57 \mathrm{Gyr}$. For this reason we consider in our analysis also different values of $S_{\mathrm{pp}}(0)$.

Including the updated OPAL EOS the best agreement between meteoritic and seismic age could be achieved with Adelberger et al.'s (1998) $S_{\mathrm{pp}}(0)=4.00 \times 10^{-25} \mathrm{MeV}$ b. Hence, by taking into account the relativistic corrections in the EOS there is no need for an artificial increase of $S_{\mathrm{pp}}(0)$, as suggested 
Table 1. Characteristic quantities of selected solar models. The indices 0 , ph, cz, and $c$ denote initial, photospheric, bottom of convective envelope, and centre, respectively. MHD-R is the abbreviation for the MHD EOS containing the relativistic corrections in $\Gamma_{1}$.

\begin{tabular}{ccccccccccccc}
\hline \hline Model & $\frac{\text { age }}{\text { Gyr }}$ & EOS & $Y_{0}$ & $Z_{0}$ & $Y_{\mathrm{ph}}$ & $Z_{\mathrm{ph}}$ & $\frac{r_{\mathrm{cz}}}{R_{\mathrm{ph}}}$ & $X_{\mathrm{c}}$ & $Y_{\mathrm{c}}$ & $\frac{\rho_{\mathrm{c}}}{\mathrm{gcm}^{-3}}$ & $\frac{T_{\mathrm{c}}}{10^{6} \mathrm{~K}}$ & $\frac{S_{\mathrm{pp}}(0)}{10^{-25} \mathrm{MeVb}}$ \\
\hline 1 & 4.58 & OPAL 01 & 0.2755 & 0.01995 & 0.2453 & 0.01805 & 0.7132 & 0.3353 & 0.6432 & 152.87 & 15.73 & 3.89 \\
2 & 4.58 & OPAL 96 & 0.2749 & 0.01995 & 0.2449 & 0.01806 & 0.7132 & 0.3289 & 0.6428 & 152.70 & 15.72 & 3.89 \\
3 & 4.60 & OPAL 01 & 0.2752 & 0.01995 & 0.2451 & 0.01805 & 0.7125 & 0.3342 & 0.6443 & 153.16 & 15.73 & 3.89 \\
4 & 4.60 & MHD-R & 0.2757 & 0.01997 & 0.2452 & 0.01805 & 0.7141 & 0.3341 & 0.6444 & 153.22 & 15.74 & 3.89 \\
5 & 5.00 & OPAL 01 & 0.2714 & 0.02013 & 0.2405 & 0.01816 & 0.7082 & 0.3133 & 0.6650 & 159.82 & 15.84 & 3.89 \\
6 & 4.58 & OPAL 01 & 0.2758 & 0.01989 & 0.2460 & 0.01803 & 0.7118 & 0.3362 & 0.6423 & 151.35 & 15.66 & 4.00 \\
\hline
\end{tabular}

by previous works, in order to obtain a better agreement between seismic and meteoritic age.

The code and physics used to compute the various solar models are described briefly in the next section, followed by the consequences for the seismic age obtained by means of the SFSA (Sect. 3). In the final part the results are discussed.

\section{The new solar models}

We computed a large number of solar models using the GARching SOlar Model (GARSOM) code which has been described in its latest version in Schlattl (2001). Our standard model has been compared with other contemporary solar models by Turck-Chièze et al. (1998), who found a good agreement between various programs.

The solar photospheric radius and luminosity have been assumed to be $695.51 \mathrm{Mm}$ (Brown \& Christensen-Dalsgaard 1998 ) and $3.8646 \times 10^{33} \mathrm{erg} / \mathrm{s}$, respectively. The surface metal ratio has been taken from Grevesse \& Noels (1993), thus $Z / X=0.0245$. The mixing length parameter (Böhm-Vitense 1958 ), initial helium and metal content have been adjusted in all models to reproduce these values with an accuracy better than $10^{-4}$.

In the actual calculations the latest OPAL-opacities (Iglesias \& Rogers 1996) completed in the low-temperature regime by tables of Alexander \& Fergusson (1994) have been implemented. The outer boundary condition was determined assuming an Eddington grey atmosphere. Microscopic diffusion of hydrogen, helium and all major metals is taken into account. For the EOS we used either the OPAL- (Rogers et al. 1996) or the MHD-tables (Hummer \& Mihalas 1988; Mihalas et al. 1988; Däppen et al. 1988). The original OPAL EOS (OPAL96) has been updated by treating electrons relativistically and by improving the activity expansion method for repulsive interactions (Rogers 2001), denoted OPAL01 in the following.

In the case of MHD EOS the relativistic corrections are not directly included in the tables. We have therefore corrected the adiabatic index $\Gamma_{1}$ employing the expression of Elliott \& Kosovichev (1998),

$\frac{\delta \Gamma_{1}}{\Gamma_{1}} \equiv \frac{\Gamma_{1, \text { rel }}-\Gamma_{1}}{\Gamma_{1}} \simeq-\frac{2+2 X}{3+5 X} \frac{k T}{m_{\mathrm{e}} c^{2}}$,

where $T$ is the temperature, $m_{\mathrm{e}}$ the electron mass, $c$ the light speed in vacuum, $k$ the Boltzmann constant, and $X$ the hydrogen mass fraction. As expected, the correction to $\Gamma_{1}$ is negative,

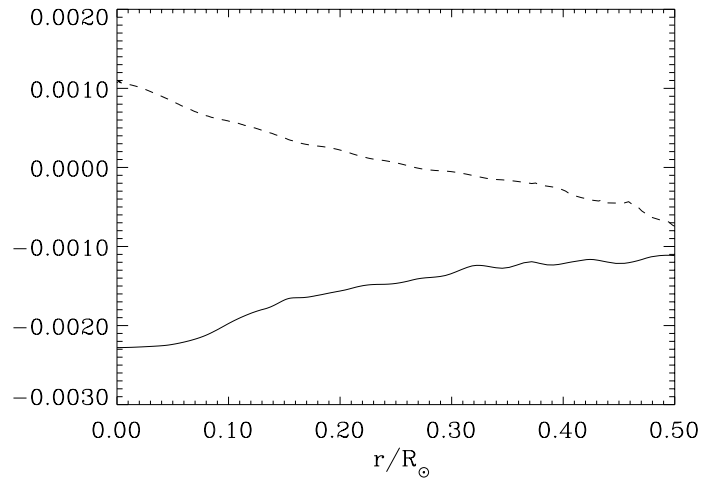

Fig. 1. The differences of $\Gamma_{1}$ (solid line) and $\rho$ (dashed) between two models which either neglect (model 1) or contain the relativistic corrections (model 2$)$ in the sense (model $2-\operatorname{model} 1) /($ model 1$)$.

since its value is $5 / 3$ for the non-relativistic and $4 / 3$ for the extremely relativistic case.

The nuclear reaction rates are taken either from Bahcall et al. (1995) or from Adelberger et al. (1998) with $S_{\mathrm{pp}}(0)$ being $3.89 \times 10^{-25} \mathrm{MeV} \mathrm{b}$ in the first and $4.00 \times 10^{-25} \mathrm{MeV} b$ in the latter case. Other differences in the reaction rates are not very significant in determining the evolutionary stage of the solar core.

\section{Results for the solar age}

We have computed solar models following the evolution from the zero-age main sequence with ages ranging from 4.40 to $5.00 \mathrm{Gyr}$ in steps of $0.1 \mathrm{Gyr}$. Some basic quantities of a selection of models are summarized in Table 1.

For the higher ages the initial helium content has to be reduced to obtain the correct solar luminosity (compare models 1 and 5). Nevertheless, a larger lifetime leads to a steeper He profile toward the centre causing a larger central $\mathrm{He}$ abundance. The consequent increase of the opacity near the core demands an higher central temperature to produce the same amount of energy. This effect is further enhanced by diffusion which is operating longer for greater ages and is further increasing the central He content. Since the relativistic correction to $\Gamma_{1}$ increases with temperature (Eq. (1)), the inclusion of relativistic effects has a larger influence on older models. The relative differences in the profiles of $\Gamma_{1}$ and the density are shown in Fig. 1. 


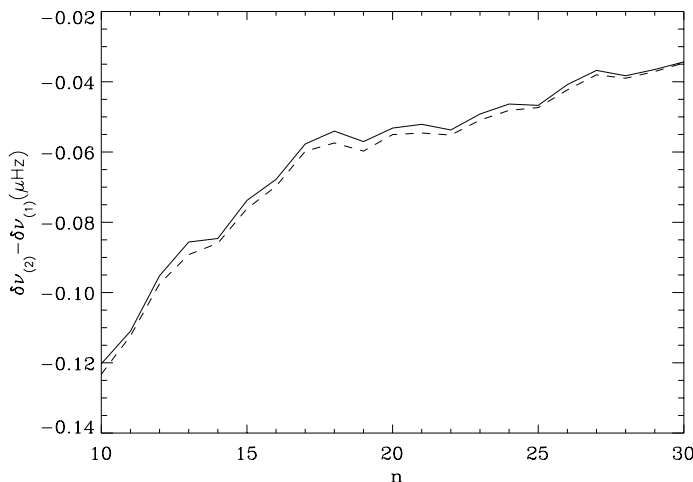

Fig. 2. The differences of the quantity $\delta v_{n, \ell}$ between two models which either neglect (model 1) or contain the relativistic correction (model 2) in the sense (model 2 - model 1) for an age of $4.20 \mathrm{Gyr}$ (solid line) and 4.70 Gyr (dashed line).

Table 2. The best-fit age and the corresponding minimum of $\chi^{2}$ for the grid with different equations of state and different values of $S_{\mathrm{pp}}(0)$ in units of $10^{-25} \mathrm{MeV} \mathrm{b}$.

\begin{tabular}{cccccc}
\hline \hline & \multicolumn{3}{c}{$\ell=0$} & \multicolumn{2}{c}{$\ell=1$} \\
EOS & $S_{\mathrm{pp}}(0)$ & $t_{\text {seis }}$ & $\chi_{0}^{2}$ & $t_{\text {seis }}$ & $\chi_{1}^{2}$ \\
\hline OPAL96 & 3.89 & $4.664 \pm 0.088$ & 1.05 & $4.672 \pm 0.088$ & 1.66 \\
OPAL01 & 3.89 & $4.584 \pm 0.088$ & 1.45 & $4.624 \pm 0.072$ & 1.66 \\
MHD & 3.89 & $4.664 \pm 0.080$ & 1.00 & $4.680 \pm 0.095$ & 1.65 \\
MHD-R & 3.89 & $4.608 \pm 0.040$ & 1.07 & $4.640 \pm 0.088$ & 1.25 \\
OPAL01 & 4.00 & $4.552 \pm 0.080$ & 1.34 & $4.584 \pm 0.080$ & 1.47 \\
\hline
\end{tabular}

Models with greater $S_{\mathrm{pp}}(0)$, but the same age, have a smaller $T_{\mathrm{c}}$ (see models 1 and 6 in Table 1), as the hydrogen burning in the core is more efficient.

In order to determine the seismic age, we calculated for all the solar models the small frequency separations $\delta v_{\ell, n}$ for $\ell=$ 0,1 and $n \gg \ell$. These values have been compared with latest GOLF/SOHO data for $\ell=0,1,2,3$, which have been obtained from long time series, and where the asymmetric line profile has been taken into account during the data reduction (Thiery et al. 2000). Only the frequencies of the mean multiplet $(m=0)$ are used, as for them the influence of rotation is smallest.

For the analysis, the $\chi^{2}$ method has been used, as in Dziembowski et al. (1999) or Schlattl et al. (1999);

$\chi_{\ell}^{2}=\frac{1}{M-m} \sum_{n=m}^{M} \frac{\left(\delta v_{\ell, n, \odot}-\delta v_{\ell, n, \text { model }}\right)^{2}}{\sigma_{\ell, n}^{2}+\sigma_{\ell+2, n-1}^{2}}$

with $M=31$ for $\ell=0$ and $M=27$ for $\ell=1$, and $m$ being 10 in both cases. It is interesting to notice that including the relativistic corrections leads to a reduction of $\delta v_{\ell, n}$ of about $0.1 \mu \mathrm{Hz}$ for low frequencies (Fig. 2).

The results for the $\chi^{2}$-values in models with different ages are shown in Figs. 3 and 4. The best-fit age given by the minimal $\chi^{2}$-value $\left(\chi_{\min }^{2}\right)$ and the error determined by the condition $\chi^{2}-\chi_{\min }^{2} \leq 1$ are summarized in Table 2 .

Regardless of whether MHD or OPAL EOS is used, the best-fit age is reduced by about $0.05-0.08$ Gyr when the relativistic corrections are included. The minimal value of $\chi_{0}^{2}$ is

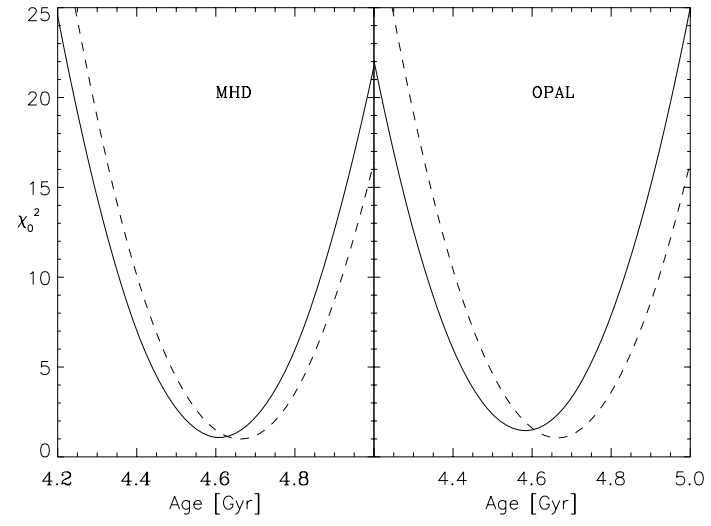

Fig. 3. $\chi_{0}^{2}$ for models with different age, neglecting (dashed line) or including the relativistic correction (solid line). The MHD-EOS has been used for the models in the left panel, the OPAL-EOS in the ones of the right panel.

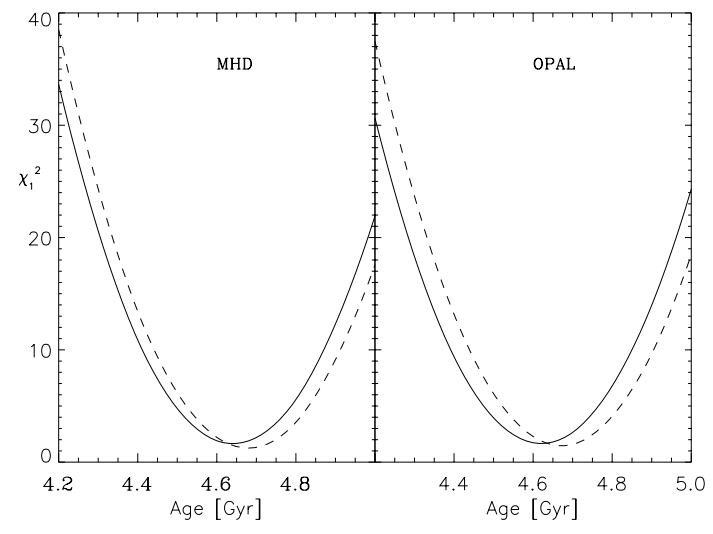

Fig. 4. Same as Fig. 3, but for $\chi_{1}^{2}$.

not significantly different for all the cases, although the models with OPAL96 EOS have a slightly smaller $\chi_{0, \text { min }}^{2}$ than those obtained with OPAL01 EOS.

It is worth noticing that with $S_{\mathrm{pp}}(0)=4.00 \times 10^{-25} \mathrm{MeV} \mathrm{b}$ the minimum $\chi^{2}$-value slightly improves for both $\ell=0$ and $\ell=1$ (Table 2). Using OPAL01 EOS, which includes the relativistic corrections in a consistent way, we obtain in this case as the best-fit age $t_{\text {seis }}=(4.57 \pm 0.11) \mathrm{Gyr}$, where we have taken the mean of the best-fit value for $\ell=0$ and $\ell=1$. This provides our most reliable value for the seismic solar age.

\section{Conclusions}

By using updated versions of the OPAL and MHD EOS the seismic age of the Sun has been redetermined using SFSA with the latest GOLF/SOHO data. The important new ingredient in both equations of state is the inclusion of the special relativistic corrections. In both cases almost the same age has been obtained.

A crucial quantity in the determination of the seismic age is the proton-proton fusion rate. With the older versions of the equations of state, a rate about $4 \%$ higher as the value of Adelberger et al. (1998) appears to be favoured, in order to obtain a better agreement between seismic and meteoritic ages. However, with the updated versions of the OPAL and MHD 
EOS the seismic age obtained with Adelberger et al.'s (1998) value for $S_{\mathrm{pp}}(0)$ is $(4.57 \pm 0.11) \mathrm{Gyr}$, which is in excellent agreement with the meteoritic age of 4.57 Gyr (Bahcall et al. 1995).

Therefore, the presently favoured value for $S_{\mathrm{pp}}(0)$ is $4.00 \times$ $10^{-25} \mathrm{MeV}$ b. However, since the uncertainties, in particular, in the opacities are supposed to be of the order of a few percent, $S_{\mathrm{pp}}(0)$ can only be determined with a similar accuracy by comparing seismic and meteoritic ages.

A further source of uncertainty is the centrifugal and magnetic distortion, but these effects can be neglected for the Sun, as discussed by Dziembowski et al.(1999).

We expect to have asteroseismic data on solar-type stars with a precision of about $0.1 \mu \mathrm{Hz}$ from future space missions or high-precision ground-based multi-site spectrographic observations. We thus think that this effect must be included in the standard modelling of solar-like stars when discussing the evolutionary changes in the stellar core.

Acknowledgements. We thank H. M. Antia for many helpful discussions. The work of H. S. is supported by a Marie Curie Fellowship of the European Community programme "Human Potential" under contract number HPMF-CT-2000-00951.

\section{References}

Adelberger, E. G., Austin, S. M., Bahcall, J. N., et al. 1998, Rev. Mod. Phys., 70, 1265
Alexander, D. R., \& Fergusson, J. W. 1994, ApJ, 437, 879

Antia, H. M., \& Chitre, S. M. 1999, A\&A, 347, 1000

Antia, H. M., Chitre, S. M., \& Thompson, M. J. 2001, A\&A, 360, 335

Bahcall, J. N., Pinsonneault, M. H., \& Wasserburg, G. J. 1995, Rev. Mod. Phys., 67, 781

Böhm-Vitense, E. 1958, Z. Astrophys., 46, 108

Bonanno, A., Murabito, A., \& Paternò, L. 2001, A\&A, 375, 1062

Brown, T. M., \& Christensen-Dalsgaard, J. 1998, ApJ, 500, L195

Däppen, W., Mihalas, D., Hummer, D. G., \& Mihalas, B. W. 1988, ApJ, 332, 261

Dziembowski, W. A., Fiorentini, G., Ricci, B., \& Sienkiewicz, R. 1999, A\&A, 343, 990

Elliott, J. R., \& Kosovichev, A. G. 1998, ApJ, 500, L199

Grevesse, N., \& Noels, A. 1993, Phys. Scr., T47, 133

Hummer, D. G., \& Mihalas, D. 1988, ApJ, 331, 794

Iglesias, C. A., \& Rogers, F. J. 1996, ApJ, 464, 943

Mihalas, D., Däppen, W., \& Hummer, D. G. 1988, ApJ, 331, 815

Rogers, F. J. 2001, Contrib. Plasma Phys., 41, 179

Rogers, F. J., Swenson, F. J., \& Iglesias, C. A. 1996, ApJ, 456, 902

Schlattl, H., Bonanno, A., \& Paternò, L. 1999, Phys. Rev. D, 60, 113002

Schlattl, H. 2001, Phys. Rev. D, 64, 013009

Tassoul, M. 1980, ApJS, 43, 469

Thiery, S., Boumier, P., Gabriel, A. H., et al. 2000, A\&A, 355, 743

Turck-Chièze, S., Basu, S., Berthomieu, G., et al. 1998, in Structure and Dynamics of the Interior of the Sun and Sun-like Stars, ed. S. Korzennik, \& A. Wilson, ESA SP-418 (ESA Publication Division, Noordwijk, The Netherlands), 555 